\title{
FORMAÇÃO EM EDUCAÇÃO FÍSICA E A SAÚDE NA ESCOLA
}

\author{
Prof. Me. Ricardo Franklin de Freitas Mussi \\ Prof. ${ }^{a}$ Ma. Denize de Azevedo Freitas \\ Prof. Me. Angelo Maurício de Amorim \\ Prof. Dr. Edio Luiz Petroski
}

\section{INTRODUÇÃO}

A gênese da Educação Física escolar (EFescolar) apresenta forte aproximação com a área da saúde. No entanto, o desenrolar histórico indica, na contemporaneidade, o relativo abandono ou mesmo a ruptura dessa relação no espaço escolarizado, em determinados contextos e/ou propostas pedagógicas.

Este ensaio discute a possibilidade de (re)aproximação da EFescolar e da saúde, sem desconsiderar as contribuições da perspectiva crítica, incluindo questões da formação profissional. A pretensão é sinalizar para o redimensionamento deste importante objeto, por considerar a EFescolar forte sustentáculo educacional para a saúde das populações, através das questões teóricas e práticas das Atividades Físicas (AF).

Em prol da popularização redimensionada da saúde na educação, tem-se que ela, antes de ser assunto médico, apresenta-se como importante questão pedagógica (BENTO apud NAHAS, 2010). A EFescolar deve, a partir do tratamento pedagógico das $\mathrm{AF}$, reconhecer suas influências biológicas, sociais, econômicas, ecológicas e históricas para a promoção da saúde. 
AF pode ser definida como movimento corporal da musculatura esquelética gerador de gasto energético (CASPERSEN; POWELL; CHRISTENSON, 1985), representando comportamento humano complexo, sendo praticada espontaneamente ou por necessidade (FONSECA; NASCIMENTO; BARROS, 2012), e influenciada por determinantes biológicos e socioculturais (NAHAS, 2010), por relações sociais, disponibilidade de equipamentos e pela mídia (MARQUES; GAYA, 1999). A escola representa o local ideal para implementação de estratégias de promoção da $\mathrm{AF}$ e da educação para a saúde (PRATES; NASCIMENTO, 2012).

A saúde deve ser reconhecida como condição humana multidimensional (física, social e psicológica), caracterizada como pertencente ao continuum com polo positivo - referente à capacidade de apreciar a vida e resistir aos desafios - e polo negativo - indicado pela morbimortalidade (BOUCHARD et al., 1990). A EFescolar deve se dedicar à compreensão de seus determinantes, planejando e implantando estratégias para o desenvolvimento de atitudes relacionadas ao polo positivo, suscitando debates e ampla prática reflexiva sobre a relação entre atividade física e saúde (AFS).

Ensinar e aprender sobre AF para a saúde visa a que as pessoas tornem-se capazes de escolher a melhor maneira para desenvolver as atividades do cotidiano, frente a representações culturais, sociais, econômicas, ambientais e biológicas.

Assim, a definição de EF, adotada neste ensaio, compreende que ela representa campo de formação, investigação e intervenção acadêmico-profissional relacionado à promoção da $\mathrm{AF}$ de sujeitos e coletividades (FONSECA et al., 2012). 


\section{A FORMAÇÃO EM EDUCAÇÃO FÍSICA E A SAÚDE NA ESCOLA: ONDE ESTAMOS?}

Discutir a docência do conhecimento sobre saúde na EF passa pelo aumento da quantidade de seus cursos de graduação, acima da média geral da área da saúde no Brasil, nas últimas décadas (HADDAD et al., 2010). Esta situação representa provável resposta à cobrança social da profissionalização para o mercado de trabalho.

O crescimento dos cursos de EF gera demanda por docentes para componentes da área da saúde, capazes de moderar sua aproximação acadêmica com as ciências sociais, humanas e biológicas (FONSECA et al., 2011), ou seja, um professor que domine o conhecimento científico, tecnológico e se aproprie das questões humanísticas e sociais (CARVALHO; CECCIM, 2006).

O docente especialista no processo de ensino -aprendizagem da saúde, neste contexto, continua sendo aquele com domínio teórico da AFS, acrescido do conhecimento das técnicas e metodologias educacionais fomentadoras da aproximação das discussões biomédicas com as humanidades e a sociologia.

Haddad et al. (2010) comentam que esse crescimento do número de cursos superiores de EF não garante boa distribuição geográfica, e que a educação e o esporte mantêm seus velhos problemas, e a saúde vem carregada de novos. Tais dificuldades devem persistir enquanto não se passar a formar professores de EF capazes de efetivar práticas de ensino em educação, esporte e saúde, sem que uma área submeta ou enviese a discussão e a prática da outra. 
Esta sugestão pretende garantir que a formação inicial em EF evite o afastamento das novas ou inovadoras discussões metodológicas e conceituais relativas à docência da saúde na EF, efetivando a EF como profissão da saúde, ainda figurando como profissão e componente curricular da educação, com os conteúdos da AFS devendo ser tratados por licenciados no ambiente escolar (FONSECA et al., 2012).

Apesar da EF ser a principal responsável pela intervenção com AF, seus conteúdos e vivências são transdisciplinares e multiprofissionais, podendo ser analisados sob a ótica das ciências naturais, sociais, das humanidades ou mesmo das ciências aplicadas, permitindo que sejam implementadas ações para sua contemplação pelas demais profissões de saúde, por intervenções diretas (Fisioterapia e Terapia Ocupacional) ou por orientação geral (demais profissões) (FONSECA et al., 2012).

Para garantir a presença redimensionada dos conteúdos relativos à AFS na EFescolar, uma questão prioritária é o processo de formação inicial ou mesmo continuada dos educadores. Se for decidido que durante as aulas de Efescolar serão trabalhadas questões fundamentais sobre AF, aptidão física e saúde, tornar-se-á igualmente relevante o aprofundamento destes conteúdos e suas metodologias de ensino durante os cursos de graduação (NAHAS, 2010).

Assim, será garantida facilitação democrática do acesso ao conhecimento que foi ou está sendo produzido (clássicos e inovações), para proporcionar ao cursista formação teórico-prática de qualidade, ainda pretendendo a autonomia na sua busca e na produção (BARBOSA-RINALDI, 2008), funções fundamentais para o desenvolvimento de EF e EFescolar de qualidade. 
Destarte, a universidade é espaço privilegiado para apropriação e difusão de conhecimento, cultura e valores sociais (função socializadora), manifestação das capacidades individuais (função orientadora), acréscimo dos conhecimentos (função pesquisadora) (ZABALZA, 2004).

Dentre as dificuldades históricas para a prática pedagógica na saúde, tem-se o início da intervenção da EF na área antes de sua solidificação, apesar de os cursos de formação inicial buscarem atender às diretrizes das políticas públicas brasileiras em seus currículos, inclusive as de saúde (BENEDETTI; SANTOS, 2012). Estudos demonstram que o perfil de formação universitária não vem alicerçando, nem na teoria, nem na prática, as futuras ações educacionais para a saúde (SOUZA; LOCH, 2011).

A formação para prática pedagógica destas questões na escola devem ter como premissas os seguintes objetivos: fomentar a discussão teórica de conteúdos relativos à AFS e temas afins; discutir sua presença nas abordagens teórico-metodológicas da Efescolar; efetivar vivências para que os estudantes percebam as especificidades e similaridades das propostas metodológicas. Neste contexto, é rechaçado o entendimento de que os professores de EF não são preparados para trabalhar conceitos, textos e práticas de maneira integrada e intencional (NAHAS, 2010).

O limitado cuidado pedagógico, nos cursos de licenciatura em EF, quanto ao ensino dos conteúdos e práticas pedagógicas na área da saúde não atendem aos desejos estudantis nem ao mercado, por consequência, nem à sociedade. Esta situação tem fomentado busca por cursos de especialização, mesmo fora da área, como na saúde coletiva, por se dedicarem a discutir e formar para a educação em saúde, na perspectiva histórico-crítico-social. 
As discussões quanto à formação docente em EF figuram como questão relevante no Brasil, desde os anos de 1980 (RANGEL-BETTI; BETTI, 1996). Contudo, dada a complexidade de seu objetivo, o que o professor deve dominar para que seja capaz de lecionar ainda representa importante problemática em sua formação (RAMOS; GRAÇA; NASCIMENTO, 2008).

As questões apresentadas indicam a necessidade de reorientação dos caminhos da EF na saúde, desde a formação inicial até a luta por melhores condições laborais, pois a superação dos problemas coletivos irá gerar melhorias contributivas para a construção da sua identidade profissional, considerando suas várias faces (BENEDETTI; SANTOS, 2012).

\section{EDUCAÇÃO FÍSICA ESCOLAR E SAÚDE: APROXIMAÇÕES E DISTANCIAMENTOS}

Para que a EFescolar brasileira se (re)aproxime ou se (re)aproprie das discussões no campo da promoção da saúde, é importante assumir que a escola é espaço fundamental para ações educacionais com esta finalidade, com esta disciplina passando a aceitar o papel central para sua condução teórico-prática intencional, centrada nas questões da AFS, evitando sua redução a mero ato técnico ou relação científica da causalidade entre AF e saúde.

Nessa perspectiva, reconhecer as bases das abordagens teórico-metodológicas da Efescolar focadas na saúde pode auxiliar no reconhecimento dos limites e das possibilidades de sucesso na ação profissional contemporânea. Tal atitude permitirá a seleção voluntária 
de conteúdos, métodos, técnicas e critérios avaliativos que legitimem a EFescolar como principal espaço educacional para a saúde dos sujeitos e da comunidade a partir das AF.

A primeira proposta da EFescolar articulada no campo da saúde foi a higienista, fruto do inovador direito à saúde do início do século XX (MOULIN, 2009). Mais que uma perspectiva pedagógica da EF, o higienismo desejou a reestruturação social nos mais diversos campos.

A proposta higienista pretendia formar um novo Brasil, uma nova sociedade e um novo homem, pelo disciplinamento dos corpos e pela adoção de hábitos de vida em prol da saúde, da paz e da harmonia social (SOARES, 2004). A EF assumiu a responsabilidade de promover AF (ginástica, esporte, jogos, recreação, exercício físico e outras) capaz de disciplinar os hábitos ligados à saúde (meramente biológica).

Esta compreensão estava fundamentada na ideia da causalidade entre AF e saúde. Naquele momento, foi aceito e determinado que apenas a incorporação da AF seria suficiente para a prevenção, o tratamento e a cura de enfermidades nas pessoas ou comunidades.

No entanto, as escolas, especialmente as públicas, não parecem estruturadas para garantir práticas regulares de $\mathrm{AF}$, propiciadoras do desenvolvimento corporal presente nessa proposta pedagógica (ALVES, 2007). Poucas destas instituições têm quadras poliesportivas, espaços para aulas práticas, quantitativo mínimo de equipamentos, número e distribuição de aulas adequadas e profissionais qualificados, situações imprescindíveis para efetivação da pedagogia higienista. 
Além disto, investigações sobre AFS em crianças identificaram que a relação entre desenvolvimento infantil e prática de AF sofre influências para além do ambiente escolar, perpassando as relações da criança com os demais ambientes e a diversidade de fatores culturais, valores e atitudes (BAUMAN; SMITH, 1999), o que impacta negativamente as bases da causalidade AF e saúde apenas pela ação escolar.

A continuidade no (re)pensar pedagógico, a partir da biologia e da EF, pretendendo uma EFescolar capaz de superar modelos tradicionais (higiênicos e eugênicos) (DARIDO, 2003), foi e vem sendo responsável pelo desenvolvimento e pela evolução de novas abordagens pedagógicas da EF na área da saúde.

A partir da década de 1990, é proposta nova abordagem pedagógica intitulada 'saúde renovada'. A pretensão era reformular a associação entre EF e saúde na escola, com novo tratamento pedagógico dos conteúdos, para adequação ao propósito de educar para a saúde, na superação do ideal exclusivamente da aptidão física. Ressalte-se que esta disciplina escolar não se dedica apenas à promoção da saúde, mas a ausência deste objetivo pode influenciar negativamente a qualidade e o sucesso de seus programas (GUEDES; GUEDES, 1997).

Confirmando esta informação, alguns indicativos regionais brasileiros sinalizam para um quadro preocupante, mas a epidemia de sedentarismo entre jovens ainda está sendo confirmada no Brasil (NAHAS; BARROS; BEM, 2004), principalmente devido à falta de estudos com design confiável e de caráter verdadeiramente representativo da população. 
A EFescolar deve ir além da promoção da $\mathrm{AF}$, de caráter fisiológico, dedicando-se à discussão dos fatores relacionados ao estilo de vida e à interferência ambiental, socioeconômica e cultural frente à promoção da saúde e ao combate dos fatores de risco para o adoecimento.

A base estruturante da 'saúde renovada' é caracterizada por cinco elementos: conceituação didático-pedagógica da saúde, análise das tendências pedagógicas de EF brasileira, determinação de objetivos e conteúdos da EFescolar para a promoção da saúde, estruturação e aplicação de avaliação morfofuncional e da aprendizagem.

O professor responsabiliza-se pelo diagnóstico e acompanhamento das múltiplas possibilidades de desenvolvimento estudantil (crescimento, composição corporal e desempenho motor), utilizando AF diversificada (jogos, brincadeiras, esportes, entre outros) em prol da aquisição e aderência de comportamentos saudáveis, para além da sala de aula (XAVIER FILHO; ASSUNÇÃO, 2005). Efetivamente, as aulas devem atingir objetivos ligados à educação para a saúde, pela seleção, organização e promoção de práticas motivadoras para o comportamento ativo e pela opção consciente de um estilo de vida saudável (GUEDES, 1999).

A adoção consciente e intencional de hábitos saudáveis, adquiridos na juventude, fundamentalmente pela oportunidade de momentos para aquisição consciente do estilo de vida positivo, fruirá de práticas pedagógicas a partir das representações da $\mathrm{AF}$ (ginástica, jogos, esportes e outras representações), intencionalmente trabalhadas para este fim. É proposta uma EFescolar disposta à formação integral do cidadão e sujeito consciente dos limites e possibilidades das práticas da AF em prol da saúde. 
Negativamente, é indicado que a 'saúde renovada' universalizou uma proposta de ação independente do nível de desenvolvimento da criança ou do adolescente, homogeneizando realidades heterogêneas (social, cultural, motora e outras), às vezes compreendendo a saúde de maneira limitada, reduzindo a importância da subjetividade, variabilidade e indefinição da atitude humana, responsabilizando o indivíduo por seu sucesso ou fracasso (XAVIER FILHO; ASSUNÇÃO, 2005).

Em momento histórico mais atualizado, a saúde coletiva, especificamente por intermédio da educação em saúde, vem apresentando contribuições pedagógicas na manipulação destes objetos, valorizando a proposta de saúde como fenômeno multidimensional e o desenvolvimento da coparticipação dos sujeitos na produção da relação saúde-doença, não presente no higienismo e pouco explorada na 'saúde renovada'.

Carvalho e Ceccim (2006) citam que o ensino da saúde, como expressão do "bem-estar físico, mental e social", deve se intercruzar com a biologia, a psicologia, as humanidades, as ciências sociais e humanas, em um reformulado projeto político-pedagógico. $\mathrm{O}$ ponto de partida da educação em saúde entende a simultaneidade de cultura, ambiente e biologia como determinantes históricos e regionais, norteadores das intervenções coletivas, coordenadas e cooperadas.

Pensar a saúde representa entendê-la como resultante de um contínuo processo de produção e reprodução de diversificadas condições geradoras do adoecimento, assim como de desenvolvimento do bem-estar, da longevidade e da qualidade de vida (QV) (MINAYO, 2006). 
A EFescolar deve, portanto, assumir-se como veículo para seu desenvolvimento pelo diálogo e pela intervenção direta na escola, com conteúdos relevantes que tematizem saúde, estilo de vida ativo e sua diversificada relação com componentes de ordem individual e coletiva, desestabilizando a relação de causalidade entre AF e saúde (DEVIDE, 2003).

\section{PERSPECTIVAS E DESAFIOS DA SAÚDE NA EDUCAÇÃO FÍSICA ESCOLAR}

Antes de pensar em resolver quais os métodos e técnicas para o ensino da AFS na EF, é importante considerar que o estudo de Silva e Malina (2000) identificou quase metade dos escolares investigados sem aulas regulares de EFescolar. Paula, Silva e Kocian (2011) citaram a falta de clareza dos conteúdos como principal causa de evasão destas aulas. Talvez sejam estes os primordiais desafios a serem resolvidos para a legitimidade e o fortalecimento dessa disciplina.

Acrescente-se, ainda, que o pouco sentido apresentado durante suas atividades vem gerando o desinteresse estudantil e a busca pela dispensa das aulas (BETTI; ZULIANI, 2002). Eles fundamentam-se, principalmente, nas permissividades previstas na lei, como, por exemplo, estarem engajados em outras práticas formais de AF.

A questão que ressurge é: seria a EFescolar apenas um momento de prática de AF? Nahas e Bem (1997) indicam, como de fundamental importância para o delineamento e a legitimação das suas funções profissionais e de pesquisa, a necessidade da identificação dos aspectos da corporeidade de responsabilidade direta e exclusiva da EF. 
Miranda (2006) apresenta a carência de estudos brasileiros que investiguem AFS no campo escolar, diferentemente daqueles desenvolvidos nos Estados Unidos, Canadá, Reino Unido e Norte Europeu, baseados no entendimento de que diversas enfermidades, especialmente as crônicas, frutificam de comportamentos inadequados após anos de vida.

Ao reconhecer que a EFescolar não representa espaço para práticas de AF, focadas apenas no desenvolvimento da aptidão física, faz-se emergencial a legitimação da sua função frente à sociedade. É importante compreendê-la como a disciplina encarregada de apresentar, discutir e vivenciar conteúdos direta ou indiretamente ligados à AFS.

Nesta perspectiva, os desafios postos são: maximização, nas aulas, da participação em atividades motoras, a partir de estratégias ágeis de transição entre atividades; melhor aproveitamento dos materiais disponíveis; informações adequadas ao nível dos escolares; valorização de atividades diversificadas que possam gerar aderência e perpetuação de suas práticas no período extraescolar (GUEDES; GUEDES, 1997).

Mesmo com professores costumeiramente citando a saúde para justificar a manutenção da EFescolar, infelizmente sua concretude costuma ser simplista e ingênua, distante da perspectiva multidimensional da promoção da saúde, corriqueira na saúde coletiva, geralmente enfocando a aptidão física (LOCH, 2011) e esquecendo as possibilidades cognitivas, culturais e sociais emergentes das práticas.

Na busca de proposta que represente atuação da EFescolar na perspectiva ampla da educação em 
saúde, foi eleito o Programa de Formação Escolar de Quebec/Canadá, por atribuir à EFescolar a função de possibilitar que o aluno "desenvolva seu agir corporal, sozinho ou em interação, aprendendo progressivamente a tomar conta de sua saúde e de seu bem-estar" (GOUVERNEMENT DU QUÉBEC, 2001, p. 252).

Mesmo considerando-os como determinação governamental canadense, apesar dos questionamentos e incertezas, os cursos de formação naquele país se adequaram para formar futuros professores de EF em sintonia com suas proposições, educação para a saúde, considerando a formação interdisciplinar pautada na ciência da educação, na cinesiologia (no caso, estudo do movimento) e nas ciências da saúde.

A expectativa é que, ao final do curso, os professores possuam saberes disciplinares ligados às ciências da AF; saberes próprios da educação para a saúde, ligados à EF; saberes pedagógicos ligados à pedagogia geral, próprios ao ensino da disciplina, relacionados à maneira de os alunos adquirirem as competências na disciplina; saberes profissionais interligando atitudes e experiências práticas no campo disciplinar, pedagógico e profissional (LÉBE, 2005).

Em síntese, esta proposta de formação centraliza as questões da educação em saúde, em que as dimensões físicas, mentais e sociais que compõem o sujeito devem ser tematizadas numa perspectiva de totalidade da compreensão humana.

Neste sentido, a EFescolar deve propiciar ao estudante o desenvolvimento da compreensão para a incorporação e a continuidade do comportamento ativo e saudável no desenvolvimento da saúde e do bem-estar 
(IBDEN, p. 20-21). As aulas, com AF diversificada deve oportunizar ao estudante desenvolver o repertório motor, estratégias cognitivas, comportamentos e atitudes capazes de promover melhor adequação à vida (LÉBE, 2005, p. 16).

O escolar deve ser capaz de compreender o sedentarismo como motivo de preocupação de saúde individual e coletiva, integrado ao estilo de vida pós-moderno, associado aos avanços e ao acesso às tecnologias redutoras do movimento corporal (ALVES, 2007). Ainda é objetivo da EFescolar que o estudante compreenda a AF distribuída nos domínios de deslocamento, atividades domésticas, trabalho e lazer, representada por ginástica, exercícios, danças, lutas e esportes (FONSECA; NASCIMENTO; BARROS, 2012).

Além de assimilar esses conceitos, o estudante deve compreender as peculiaridades da AF em sua realidade, distribuída e oportunizada de maneira desigual, segundo suas dimensões e manifestações sob determinantes históricos, econômicos, ambientais, biológicos e culturais limitantes ou estimulantes de suas práticas, assim impactando a sua saúde e QV.

A AF representa fator para $Q V$, e a EF não deve estar alheia a isto, reconhecendo as evidências e aceitando, entre seus objetivos fundamentais, os de pesquisar e intervir nas questões relacionadas à $\mathrm{AF}$ e QV de pessoas e grupos (NAHAS; BEM, 1997). A EF, na escola, não deve se furtar a esta ação, uma vez que as práticas de $\mathrm{AF}$ e esportivas costumam ser socialmente associados à saúde (STIGGER, 2002), pois sua falta justifica ações educacionais e de saúde pública (PRATES; NASCIMENTO, 2012) para a reversão do estilo de vida arriscado para a manutenção da saúde. 
Com inspiração nesta perspectiva canadense, numa eventual concepção de proposta didático-pedagógica para as aulas de EF na realidade brasileira, tomando como referências as proposições legais presentes nos Parâmetros Curriculares Nacionais (BRASIL, 1998), organizam-se os conhecimentos em três blocos: atividades rítmicas e expressivas; esportes, jogos, lutas e ginástica; conhecimento sobre o corpo.

O bloco ligado ao conhecimento sobre o corpo propõe que ele ultrapasse a instrumentalização fragmentada de conhecimentos inerentes à anatomia humana, à fisiologia do exercício e ao treinamento desportivo. A proposta deve partir destes temas particulares e transcender para o exercício de compreensão e vivência de construção de comportamentos ligados à prática de AF regular. Sua estratégia metodológica contempla os conhecimentos inerentes aos outros blocos estruturados de acordo com a seguinte proposição:

- os objetivos das aulas devem versar sobre aprendizagem motora, questões teóricas e conceituais relativas às dimensões e representações das AF;

- os conteúdos devem focar as origens, momento histórico contemporâneo e processo evolutivo, suas normas e características específicas, seus gestos técnicos básicos, as estratégias individuais e coletivas para participação, diferenças culturais e densidades regionais, relações ambientais, possibilidades competitivas e participativas, modificações orgânicas (fisiológicas e cinesiológicas) e benefícios das AF para a saúde; 
- as estratégias avaliativas devem buscar o diagnóstico e acompanhamento morfofuncional, teórico e motor, sendo capazes de verificar as aprendizagens multidimensionais relacionadas às práticas da $\mathrm{AF}$ (ginásticas, exercícios, danças, lutas e esportes), valorizando a exploração dos espaços e equipamentos disponíveis;

- as aulas devem desenvolver AF contextualizadas ao desenvolvimento motor, cognitivo, compreensivo e de aptidão física em prol da participação individual e coletiva. Antes, durante e após as práticas, devem ser valorizados os aspectos técnicos, táticos, físicos e teóricos das representações das $\mathrm{AF}$, focando o enriquecimento motor, o desenvolvimento da consciência corporal (orgânica e psicológica) frente a diferentes graus de exigência física e cognitiva na resolução de problemas.

A concretização desta proposta viabiliza a indissociabilidade das questões cognitivas, motoras e orgânicas para o desenvolvimento da saúde pela aprendizagem das AF. Sinaliza para o reconhecimento da aprendizagem motora diversificada, com componentes técnicos e táticos, individuais e coletivos, e a percepção das alternâncias corporais a partir da intencionalidade pedagógica, capaz de fomentar o empoderamento dos participantes quanto à opção e à crítica de caminhos e desafios para a manutenção e o desenvolvimento da saúde, individual e coletiva, durante e após o período escolar, a partir da EFescolar, suas especificidades, objetos e objetivos educacionais. 
Fundamentalmente as aulas devem representar a compreensão de uma saúde resultante da ação protetora da pessoa e da sociedade, conhecedora e superadora dos riscos do adoecimento, em prol do estilo de vida saudável e, portanto, promotora da integração humana no socioecossistema (MINAYO, 2006). O desafio posto é que o professor atue na educação em saúde, considerando os saberes técnicos, acadêmicos e políticos, a partir de seu campo específico, no caso, as intervenções intencionais pela AF na EFescolar.

\section{CONSIDERAÇÕES FINAIS}

A capacidade de abordar, com intencionalidade pedagógica, as questões da AFS caracteriza e individualiza a atuação profissional da EF frente às demais profissões da área das ciências da saúde, especialmente no campo escolar.

Neste sentido, os professores de EF devem ser formados dentro de uma proposta orientada para a aproximação e o aprofundamento teórico-prático das questões multidimensionais da AF e saúde, considerando as possibilidades e limitações desta relação presentes nas abordagens pedagógicas, constituídas historicamente para nortear sua intervenção profissional.

Efetivamente, a EFescolar deve se responsabilizar pelo desenvolvimento de práticas e discussões contextualizadas e intencionais, considerando a AF, suas dimensões e representações como elemento importante para a promoção da saúde e prevenção de doenças.

As aulas devem promover a interação das questões teórico-práticas da $\mathrm{AF}$ e da saúde a partir de 
esportes, manifestações culturais, danças, ginásticas, lutas, exercícios físicos e demais expressões e, ainda, considerando as questões sociais, culturais, econômicas, ambientais, biológicas e psicológicas relacionadas, direta ou indiretamente, como fomentadoras ou limitantes de sua prática.

O que não deve nem pode ocorrer é a negação dos conteúdos da AFS na EFescolar, pois esta situação significaria negar a própria essência do processo educativo pautado na disponibilização de conhecimento acumulado, social e historicamente, pela humanidade, e a reflexão sobre e a partir destes conhecimentos, impedindo a continuidade do desenvolvimento humano e o avanço crítico-científico.

\section{REFERÊNCIAS}

ALVES, U. S. Não ao sedentarismo, sim à saúde: contribuições da Educação Física escolar e dos esportes. $\mathbf{O}$ Mundo da Saúde, v. 31, n. 4, p. 464-469, 2007.

BARBOSA-RINALDI, I. P. Formação inicial em Educação Física: uma nova epistemologia da prática docente. Movimento, v. 14, n. 03, p. 185-207, 2008.

BAUMAN, A.; SMITH, B. Geographical influences upon physical activity participation: Evidence of a "coastal effect". Australian and New Zeland Journal of Public Health, v. 23, n. 3, p.322-324, 1999. 
BENEDETTI, T. R. B.; SANTOS, S. F. da S. dos. Educação Física no contexto da saúde. In: NASCIMENTO, J. V. do; FARIAS, G. O. (org.). Construção da identidade profissional em Educação Física: da formação à intervenção. Florianópolis: UDESC, 2012.

BETTI, M.; ZULIANI, L. R. Educação Física Escolar: uma proposta de diretrizes pedagógicas. Revista Mackenzie de Educação Física e Esporte, v. 1, n. 1, p. 73-81, 2002.

BOUCHARD, C.; SHEPHARD, R. J.; STEPHENS, T.; SUTTON, J. R.; McPHERSON, B. D. Exercise, fitness and health: the consensus statement. In: (org.). Exercise, fitness and health. A consensus of current knowledge. Champaign: Human Kinetics Publishers, 1990.

BRASIL. Parâmetros Curriculares Nacionais. Brasília, DF: MEC, 1998.

CARVALHO, Y. M.; CECCIM, R. B. Formação e Educação em Saúde: aprendizados com a Saúde Coletiva. In: CAMPOS, G. W. S.; MINAYO, M. C. S.; AKERMAN, M.; DRUMOND, M.; CARVALHO, Y. M. (orgs.). Tratado de Saúde Coletiva. São Paulo: Hucitec; Rio de Janeiro: Fiocruz, 2006.

CASPERSEN, C. J.; POWELL, K. E.; CHRISTENSON, G. M. Physical activity, exercise, and physical fitness: definitions and distinctions for health-related research. Public Health Reports, v. 100, n. 2, p. 126-31, 1985.

DARIDO, S. C. Educação Física na Escola: questões e reflexões. Rio de Janeiro: GUANABARA KOOGAN, 2003. 
DEVIDE, F. P. Educação Física Escolar como via de Educação para a Saúde. In: BAGRICHEVSKY, M.; PALMA, A.; ESTEVÃO, A. (org.). A saúde em debate na Educação Física. Blumenau: Edibes, 2003.

FONSECA, S. A.; MENEZES, A. S.; FEITOSA, W. M. N.; LOCH, M. R. Notas preliminares sobre a Associação Brasileira de Ensino da Educação Física para a Saúde ABENEFS. Caderno FNEPAS, v. 2, p. 38-48, 2012.

FONSECA, S. A.; MENEZES, A. S.; LOCH, M. R.; FEITOSA, W. M. N.; NAHAS, M. V.; NASCIMENTO, J. V. Pela criação da Associação Brasileira de Ensino da EF para a saúde: Abenefs. Revista Brasileira de Atividade Física \& Saúde, v. 16, n. 4, p. 283-88, 2011.

FONSECA, S. A.; NASCIMENTO, J. V.; BARROS, M. V. G. de. A formação inicial em Educação Física e a intervenção profissional no contexto da saúde: desafios e proposições. In: NASCIMENTO, J. V. do; FARIAS, G. O. (org.). Construção da identidade profissional em Educação Física: da formação à intervenção. Florianópolis: UDESC, 2012.

GOUVERNEMENT DU QUÉBEC. La formation à l'enseignement. Les orientations. Les compétences professionalles. Québec: Ministère de l'Éducation, 2001.

GUEDES, D. P. Educação para a Saúde mediante programas de Educação Física Escolar. Motriz, v. 5, n. 1, p. 10-14, 1999.

GUEDES, D. P.; GUEDES, J. E. R. P. Características dos Programas de Educação Física Escolar. Revista Paulista de Educação Física, v. 11, n. 1, p. 49-62, 1997. 
HADDAD, A. E.; MORITA, M. C.; PIERANTONI, C. R.; BRENELLI, S. L.; PASSARELLA, T.; CAMPOS, F. E. Formação de profissionais de saúde no Brasil: uma análise no período de 1991 a 2008. Revista de Saúde Pública, v. 44, n. 3, p. 383-93, 2010.

LÈBE, R. Reflexões sobre o novo programa de Educação Física da Universidade de Montreal. In: BORGES, C; DESBIENS, J. F. Saber, formar e intervir para uma Educação Física em mudança. Campinas: Autores Associados, 2005.

LOCH, M. R. A promoção da atividade física na escola: um difícil e necessário desafio. Revista Brasileira de Atividade Física \& Saúde, v. 16, n. 1, p. 76-77, 2011.

MARQUES, A. T.; GAYA, A. Atividade física, aptidão física e educação para a saúde: estudos na área pedagógica em Portugal e no Brasil. Revista Paulista de Educação Física, v. 13, n. 1, p. 83-110, 1999.

MINAYO, M. C. S. Saúde como responsabilidade cidadã. In: BAGRICHEVSKY, M.; PALMA, A.; ESTEVÃO, A.; DA ROS, M. (org.). A Saúde em debate na Educação Física. Blumenau: Nova Letra, 2006. v. 2.

MIRANDA, M. J. Educação Física e saúde na escola. Estudos, v. 33, n. 7/8, p. 643-653, 2006.

MOULIN, A. M. O corpo diante da medicina. In: CORBIN, A.; COURTINE, J.; VIGARELLO, G. (org.). História do corpo: as mutações do olhar: o século XX. 3 ed. Petrópolis: Vozes, 2009. 
NAHAS, M. V. Atividade Física, Saúde e Qualidade de Vida: conceitos e sugestões para um estilo de vida ativo. 5ed. rev. e atual. Londrina: Midiograf, 2010.

NAHAS, M. V.; BARROS, M. V. G.; BEM, M. F. L. de. Promoção da saúde nos programas de Educação Física: educação para um estilo de vida ativo. In: LEBRE, E.; BENTO, J. (org.). Professor da Educação Física: ofícios da profissão. Porto: FCDEF, 2004.

NAHAS, M. V.; BEM, M. F. L. de. Perspectivas e tendências da relação teoria e prática na Educação Física. Motriz, v. 3, n. 2, p. 73-79, 1997.

PAULA, T.; SILVA, J.; KOCIAN, R. C. A participação nas aulas de Educação Física Escolar de Ensino Médio. Coleção Pesquisa em Educação Física, v. 10, n. 6, p. 33-40, 2011.

PRATES, M. E. F.; NASCIMENTO, J. V. do. Contribuição da disciplina qualidade de vida para a mudança de comportamento para a prática de atividades físicas em estudantes universitários. In: FOLLE, A.; FARIAS, G. O. (org.). Educação Física: prática pedagógica e trabalho docente. Florianópolis: UDESC, 2012.

RAMOS, V.; GRAÇA, A. B. dos S.; NASCIMENTO, J. V. do. O conhecimento pedagógico do conteúdo: estrutura e implicações à formação em educação física. Revista Brasileira de Educação Física e Esporte, v. 22, n. 2, p. 161-71, 2008.

RANGEL-BETTI, I. C.; BETTI, M. Novas perspectivas na formação profissional em Educação Física. Motriz, v. 2, n. 1, p. 10-15, 1996. 
SILVA, R. C. R.; MALINA, R. M. Nível de atividade física em adolescentes do Município de Niterói, Rio de Janeiro, Brasil. Cadernos de Saúde Pública, v. 16, n. 4, p. 1091-7, 2000.

SOARES, C. L. Educação Física: raízes européias e Brasil. 3 ed. Campinas: Autores Associados, 2004.

SOUZA, S. C.; LOCH, M. R. Intervenção do profissional de Educação Física nos Núcleos de Apoio à Saúde da Família em municípios do Norte do Paraná. Revista Brasileira de Atividade Física \& Saúde, v. 16, n. 1, p. 5-10, 2011.

STIGGER, M. P. Esporte, lazer e estilo de vida: um estudo etnográfico. Campinas: Autores Associados, 2002. XAVIER FILHO, L. P.; ASSUNÇÃO, J. R. Educação Física. Rio de Janeiro: UNIT, 2005.

ZABALZA, M. O ensino universitário: seu cenário e seus protagonistas. Porto Alegre: Artmed, 2004. 